\title{
Estudio de los melanomas acrales en el distrito sur de Sevilla, España
}

\section{Study of acral melanomas in the southern district of Seville, Spain}

\author{
José R. Mazuecos-Gutiérrez, Isabel Ma Coronel-Pérez, José Mazuecos-Blanca y \\ Jerónimo Escudero-Ordóñez* \\ Servicio de Dermatología, Hospital Universitario Nuestra Señora de Valme, Sevilla, España
}

\begin{abstract}
Resumen
Introducción: El melanoma acral (MA) representa 5\% a 10\% de los melanomas y es el subtipo más frecuente en las poblaciones no caucásicas. Objetivos: Establecer las características de los pacientes con MA desde que se constituyó la Unidad de Lesiones Pigmentarias. Material y métodos: Se ha realizado un estudio retrospectivo de los pacientes con MA desde 2008 hasta 2018 en el Distrito Sur de Sevilla. Resultados: Se han diagnosticado 15 MA (4.2\%) de un total de 357 melanomas cutáneos, 8 en mujeres y 7 en varones, con una edad media de 58.8 años (intervalo, 24 a 92 años). Diez casos se localizaron en la planta del pie (66.7\%), cinco en la uña del primer dedo (33.3\%), dos en las manos (40\%) y tres en los pies (60\%). Hubo 11 casos (73.3\%) de melanoma lentiginoso acral, 2 (13.3\%) de melanoma de extensión superficial y otros dos de melanoma nodular. Dos pacientes se identificaron con estadio 0 (13.3\%), 8 con estadio I (53.3\%), 3 con estadio II (20\%) y 2 con estadio III (13.3\%). La media del índice de Breslow fue de $1.45 \mathrm{~mm}$ (intervalo, 0 a $5.5 \mathrm{~mm}$ ). Dicha media fue de $0.47 \mathrm{~mm}$ en los melanomas de uñas y de $1.93 \mathrm{~mm}$ en los plantares $(p<0.05)$. Los niveles de Clark fueron también más bajos en los melanomas ungueales $(p=0.077)$. Conclusiones: Esta serie ha presentado unos parámetros clínico-histopatológicos con un pronóstico bueno o aceptable y es mejor en los melanomas ungueales que en los plantares.
\end{abstract}

Palabras clave: Melanoma acral. Melanoma plantar. Melanoma ungueal.

\section{Abstract}

Introduction: Acral melanoma (AM) is a subtype of cutaneous melanoma that represents $5-10 \%$ of all melanoma cases and is the most frequent subtype in non-Caucasian populations. Objectives: To establish the clinical-pathological characteristics of patients with AM since our Pigmentary Lesions Unit was established. Material and method: A retrospective study of a series of data of patients with AM from 2008 to 2018 has been carried out. Results: 15 cases of AM (4.2\%) have been diagnosed out of a total of 357 cutaneous melanomas. There were 8 female and 7 male patients, with a mean age of 58.8 years (range between 24 and 92 years). 10 cases have been diagnosed in the sole of the foot (66.7\%) and 5 cases in the nail unit (33.3\%), two in the thumb and three in the hallux. There were 11 cases (73.3\%) of acral lentiginous melanoma, 2 cases (13.3\%) of superficial spreading melanoma and 2 cases of nodular melanoma. There were 2 patients with stage $0(13.3 \%), 8$ patients with stage I (53.3\%), 3 patients with stage II (20\%) and 2 patients with stage III (13.3\%). The mean Breslow index was $1.45 \mathrm{~mm}$ (range from 0 to 5.5 $\mathrm{mm})$. This mean was $0.47 \mathrm{~mm}$ in nail melanomas and $1.93 \mathrm{~mm}$ in plantar melanomas $(p<0.05)$. Clark levels were also lower in nail melanomas than plantar melanomas $(p=0.077)$. Conclusions: Our case series has presented clinical-histopathological parameters with a good or acceptable prognosis, being better in nail melanomas than in plantar melanomas.

Key words: Acral melanoma. Plantar melanoma. Subungual melanoma.

\footnotetext{
Correspondencia:

Fecha de recepción: 11-06-2020

Fecha de aceptación: 24-07-2020

E-mail: jeesor@gmail.es

DOI: 10.24875/MCUT.20000060

0210-5187/@ 2020 Colegio Ibero Latinoamericano de Dermatología A.C. (CILAD). Publicado por Permanyer. Este es un artículo open access bajo la licencia CC BY-NC-ND (http://creativecommons.org/licenses/by-nc-nd/4.0/).

Disponible en internet: 14-12-2020 Med Cutan Iber Lat Am. 2020;48(2):100-105 www.MedicinaCutanealLA.com
un artículo open access bajo la
} 


\section{Introducción}

El melanoma acral (MA) es un subtipo raro de melanoma cutáneo incluido dentro del grupo de los melanomas no relacionados de forma consistente con el daño solar acumulativo y que se localiza en las plantas de los pies (con más frecuencia), las palmas de las manos y las uñas 1 .

Representa $5 \%$ a $10 \%$ de todos los casos de melanoma y es el subtipo más común en las poblaciones no caucásicas, como las asiáticas, las africanas y las latinoamericanas, debido en particular a que en ellas hay una menor incidencia de melanomas vinculados con el daño solar acumulativo bajo o alto'. En estas poblaciones, el MA puede variar desde $40 \%$ hasta $70 \%^{2}$ mientras que en la raza blanca representa menos del $10 \%$, por lo general sólo un $2 \%$ a $5 \%$ de todos los melanomas. En raras ocasiones puede ser amelanótico ${ }^{3}$.

De igual modo, el melanoma ungueal (MU) se desarrolla en las uñas de manos y pies, y su localización más común se halla en los primeros dedos, quizás por el mayor tamaño de su matriz ungueal. Esta localización también es habitual en fototipos altos (20\%-30\% de los melanomas en las razas negra o asiática) y rara en la raza blanca, en la que representa $2 \%$ a $3 \%$ de los melanomas 4 .

Es más frecuente que el MA se desarrolle en pacientes adultos 0 ancianos. La incidencia aumenta en la quinta a séptima décadas, con una edad media de 65 años. No parece que tenga un claro predominio por género ${ }^{5}$. En términos histopatológicos, sólo un $70 \%$ de Ios MA corresponde a melanoma lentiginoso acral (MLA), en tanto que el resto corresponde a melanoma de extensión superficial (MES) o melanoma nodular $(\mathrm{MN})^{6}$.

La causa del MA no es clara, ya que no se correlaciona con la exposición al sol ni con las quemaduras solares; se ha sugerido que intervienen las alteraciones genéticas y los traumatismos crónicos y repetitivos $^{5}$. Por otro lado, su desarrollo no se ha vinculado con la preexistencia de nevos melanocíticos, por lo que iniciaría de novo ${ }^{3}$.

El pronóstico suele ser malo, debido a una tendencia al diagnóstico tardío pero quizás también a diferencias intrínsecas sustanciales, como una evolución biológica más agresiva respecto de los otros subtipos de melanoma ${ }^{7}$.

El objetivo de este estudio es determinar las características clínico-patológicas de los pacientes con diagnóstico de MA desde que se constituyó la Unidad de Lesiones Pigmentarias del hospital de los autores en el año 2008.

\section{Material y métodos}

Se ha realizado un estudio de cohortes, observacional y retrospectivo de una serie de datos demográficos, clínicos e histopatológicos de los pacientes con melanoma acral diagnosticados entre 2008 y 2018 en el Hospital Universitario Nuestra Señora de Valme de Sevilla.

En la tabla 1 se exponen las variables descriptivas clínicas y dermatopatológicas de todos los casos. El estadio tumoral se definió de acuerdo con la octava edición del AJCC (American Joint Committee on Cancer $)^{8}$. También se compararon las posibles diferencias en función del sexo de los pacientes y la localización del melanoma en la planta de los pies o las uñas.

Las variables se compararon con base en el tamaño y normalidad de la muestra con las pruebas $x^{2}$ y exacta de Fisher para las variables cualitativas y $U$ de MannWhitney para las variables cuantitativas. Los resultados aparecen expresados como media y desviación estándar y mediana y rango intercuartílico en las variables cuantitativas y como valor absoluto y frecuencia en las cualitativas. Se calculó la supervivencia mediante la prueba de Kaplan-Meier tras comparar los grupos con la prueba log rank. Se consideró significativo un valor de $p<0.05$.

Los datos obtenidos se introdujeron en una base de datos y se analizaron con el paquete estadístico SPSS versión 22.0 para Windows.

\section{Resultados}

Se han diagnosticado 15 casos de MA desde el año 2008 hasta el 2018 en el Área de Gestión Sanitaria Sur de Sevilla sobre un total de 357 melanomas, lo que corresponde al $4.2 \%$ de los pacientes, con una prevalencia de 4.15 por cada 100,000 habitantes. Los datos obtenidos de estos sujetos se describen en la tabla 1.

Se ha identificado a 8 mujeres $(53.3 \%$ ) y 7 varones (46.7\%), con una edad media al diagnóstico de 58.8 años, una mediana de 55 años y un intervalo de 24 a 92 años. Se han diagnosticado 10 casos en la planta del pie $(66.7 \%)$ y 5 casos en las uñas $(33.3 \%)$, todos en los primeros dedos, 2 en las manos (40\%) y 3 en los pies (60\%).

De acuerdo con la distribución por fototipos de los pacientes, 1 paciente tuvo fototipo I (6.7\%), 5 fototipo II (33.3\%), 5 fototipo III (33.3\%) y 4 fototipo IV (26.6\%).

En relación con las formas clínicas de presentación en los 10 pacientes de MA de los pies, 8 (80\%) mostraron crecimiento de lesión pigmentada, un hallazgo 
Tabla 1. Descripción de las variables de los 15 pacientes con melanoma acral

\begin{tabular}{|c|c|}
\hline Variables & $n=15$ \\
\hline $\begin{array}{l}\text { Edad al diagnóstico (años) } \\
\text { Media } \pm \mathrm{DE} \text { (intervalo) } \\
\text { Mediana (RIO) }\end{array}$ & $\begin{array}{c}58.8 \pm 18.2(24-92) \\
55(52,72)\end{array}$ \\
\hline $\begin{array}{l}\text { Sexo }[n(\%)] \\
\text { Mujer } \\
\text { Varón }\end{array}$ & $\begin{array}{l}8(53.3) \\
7(46.7)\end{array}$ \\
\hline $\begin{array}{l}\text { Fototipo [n (\%)] } \\
\text { I } \\
\text { II } \\
\text { III } \\
\text { IV }\end{array}$ & $\begin{array}{l}1(6.7) \\
5(33.3) \\
5(33.3) \\
4(26.7)\end{array}$ \\
\hline $\begin{array}{l}\text { Localización [n (\%)] } \\
\text { Planta del pie } \\
\text { Uña }\end{array}$ & $\begin{array}{l}10(66.7) \\
5(33.3)\end{array}$ \\
\hline $\begin{array}{l}\text { Forma de presentación [n (\%)] } \\
\text { Crecimiento lesión pigmentada } \\
\text { Cambio de color de uña } \\
\text { Cambio en nevo previo } \\
\text { Destrucción uña y pigmentación posterior } \\
\text { Hallazgo en exploración física } \\
\text { Lesión pigmentada ulcerada sangrante }\end{array}$ & $\begin{array}{l}7(46.7) \\
2(13.3) \\
1(6.7) \\
1(6.7) \\
2(13.3) \\
2(12.3)\end{array}$ \\
\hline $\begin{array}{l}\text { Tratamiento [n (\%)] } \\
\text { Amputación } \\
\text { Injerto } \\
\text { Sutura directa }\end{array}$ & $\begin{array}{l}3(20.0) \\
9(60.0) \\
3(20.0)\end{array}$ \\
\hline $\begin{array}{l}\text { Subtipo histológico [n (\%)] } \\
\text { MLA } \\
\text { MES } \\
\text { MN }\end{array}$ & $\begin{array}{l}11(73.3) \\
2(13.3) \\
2(13.3)\end{array}$ \\
\hline $\begin{array}{l}\text { Índice de Breslow (mm) } \\
\text { Media } \pm \text { DE (intervalo) } \\
\text { Mediana [RIO] }\end{array}$ & $\begin{array}{c}1.67(0-5.5) \\
0.62(0.0,2.0)\end{array}$ \\
\hline $\begin{array}{l}\text { Ulceración [n (\%)] } \\
\text { No } \\
\text { Sí }\end{array}$ & $\begin{array}{l}10(66.7) \\
5(33.3)\end{array}$ \\
\hline $\begin{array}{l}\text { Nivel de Clark [n (\%)] } \\
\text { I } \\
\text { II } \\
\text { III } \\
\text { IV } \\
\text { V }\end{array}$ & $\begin{array}{l}2(13.3) \\
4(26.7) \\
4(26.7) \\
3(20.0) \\
2(13.3)\end{array}$ \\
\hline $\begin{array}{l}\text { Mitosis [n (\%)] } \\
\quad<1 \\
\geq 1 \\
\text { No disponible }\end{array}$ & $\begin{array}{l}6(40.0) \\
5(33.3) \\
4(26.7)\end{array}$ \\
\hline $\begin{array}{l}\text { Invasión vascular [n (\%)] } \\
\text { No } \\
\text { Sí } \\
\text { No disponible }\end{array}$ & $\begin{array}{c}10(66.7) \\
1(6.7) \\
4(26.7)\end{array}$ \\
\hline $\begin{array}{l}\text { Invasión perineural [n (\%)] } \\
\text { No } \\
\text { Sí } \\
\text { No disponible }\end{array}$ & $\begin{array}{c}10(66.7) \\
1(6.7) \\
4(26.7)\end{array}$ \\
\hline
\end{tabular}

Tabla 1. Descripción de las variables de los 15 pacientes con melanoma acral (Continuación)

\begin{tabular}{|l|c|}
\hline Variables & $\mathbf{n}=\mathbf{1 5}$ \\
\hline Estadio [n (\%)] & \\
\hline 0 & $2(13.3)$ \\
IA & $6(40.0)$ \\
IB & $2(13.3)$ \\
IIA & $1(6.7)$ \\
IIB & $1(6.7)$ \\
IIC & $1(6.7)$ \\
IIIA & $1(6.7)$ \\
IIIC & $1(6.7)$ \\
\hline BSGC [n (\%)] & \\
Negativa & $8(53.3)$ \\
Positiva & $3(20.0)$ \\
No realizada & $5(33.3)$ \\
\hline Recidiva [n (\%)] & \\
No & $12(80.0)$ \\
Sí & $2(13.3)$ \\
Progresión & $1(6.7)$ \\
\hline
\end{tabular}

exploratorio (10\%) y otro caso con una lesión pigmentada ulcerada (10\%), en tanto que en el MU se reconocieron dos cambios en el color de la uña (40\%), un hallazgo exploratorio (20\%), una destrucción ungueal con pigmentación periungueal $(20 \%)$ y una lesión pigmentada ulcerada (20\%).

En el plano dermatopatológico, se diagnosticaron 11 casos de MLA (73.3\%), 2 de MES (13.3\%) y 2 de MN (13.3\%). De los $11 \mathrm{MLA}, 7$ se encontraron en la planta de los pies (63.7\%) y 4 en las uñas (36.3\%). Los 2 MN se localizaron en la planta del pie y, de los 2 MES, uno en la planta del pie y otro en la uña del primer dedo de la mano izquierda.

La media del índice de Breslow fue de $1.45 \mathrm{~mm}$, con límites de 0 a $5.5 \mathrm{~mm}$ y la mediana fue de $0.62 \mathrm{~mm}$. La media del grosor de Breslow de los MLA fue de 0.84 $\mathrm{mm}$, la de los MES de $1 \mathrm{~mm}$ y la de los MN de 5.25 $\mathrm{mm}$. Hubo $2 \mathrm{MA}$ in situ (13.3\%) en las uñas, 6 MA con menos de $1 \mathrm{~mm}(40 \%), 5 \mathrm{MA}$ entre 1 y $2 \mathrm{~mm}(33.3 \%)$ y 2 MA con un Breslow mayor de 2 (13\%). El nivel de Clark de los pacientes fue de 2 casos con nivel I (13.3\%), 4 casos con nivel II (26.7\%), 4 casos con nivel III $(27.7 \%)$, 3 casos con nivel IV $(20 \%)$ y 2 casos con nivel V $(13.3 \%)$. Se identificaron sólo 5 casos con ulceración (33.3\%), 3 en la planta del pie y 2 en la uña. Se dispuso de información sobre mitosis en 11 pacientes; 6 pacientes con $<1 / \mathrm{mm}^{2}(54.5 \%)$ y $5 \mathrm{con} \geq 1 / \mathrm{mm}^{2}(45.5 \%)$.

La biopsia selectiva del ganglio centinela (BSGC) se realizó en 11 pacientes (73.3\%), aunque ésta no se indicó en los dos pacientes con estadio 0 y en otros dos con estadio IA. Dicha BSGC fue positiva en 3 pacientes (27.3\%), a los que se les administró 


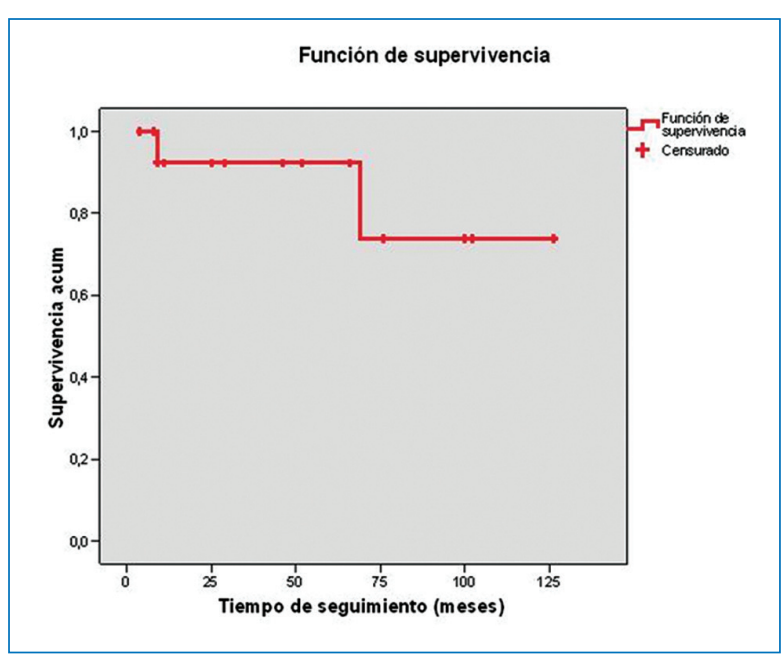

Figura 1. Supervivencia de los 15 pacientes con melanoma acral.

tratamiento adyuvante con interferón $\alpha-2 b$. Con respecto al estadio tumoral, 2 pacientes tenían estadio 0 (13.3\%), 8 estadio I (53.3\%), 3 estadio II (20\%) y 2 estadio III (13.3\%).

En el tratamiento se practicó la extirpación quirúrgica de la lesión con sutura directa en 3 pacientes $(20 \%)$ y con cierre mediante injerto de piel total en $9(60 \%)$. Se realizó la amputación digital en 3 pacientes $(20 \%)$, una en mano y dos en pies. Un paciente con MU del pie experimentó recidiva y se llevó a cabo después una desarticulación de la falange distal.

En el análisis estadístico no se hallaron diferencias significativas en función del género de los pacientes. En cuanto a la localización de los MA, la media del índice de Breslow en las uñas fue de $0.47 \mathrm{~mm}$, mientras que en la planta del pie fue de $1.93 \mathrm{~mm}(\mathrm{p}<0.05)$ y los niveles de Clark fueron predominantemente más bajos en las uñas que en los pies $(p=0.077)$. En el resto de las variables no se encontraron diferencias significativas.

Se produjeron 2 defunciones (13.3\%) en el seguimiento de los pacientes, por lo que la supervivencia del estudio fue del $92.3 \%$ al año y del $73.8 \%$ a los cinco años (Figura 1), sin diferencias en función del género $(p=0.179)$ ni la localización $(p=0.420)$.

\section{Discusión}

El porcentaje de MA en este estudio fue del $4.2 \%$, lo que se corresponde con los estudios en la raza blanca, que suelen ser inferiores al $10 \%$ de todos los melanomas $^{9}$. Este tipo de melanoma tiene una predisposición racial por los asiáticos ${ }^{10,11}$ y los sujetos de piel oscura, como afroamericanos y latinoamericanos ${ }^{2,6}$. Hay estudios de México ${ }^{12}$, Colombia ${ }^{13}$, Chile ${ }^{14}$, Puerto Rico, Perú $^{15}$ o Brasil $^{16}$ que lo confirman; sin embargo, en Argentina y Uruguay las localizaciones son similares a las de la población caucásica ${ }^{17}$. En esta serie se identificó a 6 pacientes con fototipos bajos (40\%) y sólo 4 con fototipo IV (26.6\%), y no se reconoció a ninguno con fototipos muy altos (V-VI). Tampoco se identificó ningún melanoma amelanótico; al parecer, los sujetos de piel clara son más propensos a desarrollar estos melanomas amelanóticos ${ }^{3}$.

En general, todos los estudios refieren que los MA suelen ser gruesos al momento del diagnóstico y se relacionan con una supervivencia más pobre ${ }^{9,11,16}$. Ese pronóstico desfavorable probablemente se deba al retraso del diagnóstico de los melanomas acrales en comparación con otras localizaciones anatómicas. Se ha indicado que el notorio retraso en el diagnóstico del MA se debe tanto al paciente como al médico, lo que es atribuible en parte a una falta de reconocimiento de los signos de alarma en lo que concierne al paciente y al personal sanitario. Por ello se recomienda que las campañas preventivas del melanoma sean más enfáticas en la posible afectación de las palmas, y sobre todo las plantas, para mejorar el diagnóstico temprano del melanoma en todos los sitios del cuerpo humano ${ }^{18}$.

Del mismo modo, el retraso con el que suelen diagnosticarse los melanomas ungueales, especialmente los localizados en los pies, demuestra que el MU es también una tarea pendiente de la asistencia primaria, por lo que algunos autores españoles indican que es necesaria una mejor concientización de la población y una mejor formación de los profesionales para conseguir un diagnóstico más temprano de esta entidad ${ }^{19}$.

Al contrario que lo referido con anterioridad, en esta serie diversos datos revelan un buen pronóstico de los pacientes. La gran mayoría de los MA era delgada, con un grosor medio de $1.45 \mathrm{~mm}$ y una mediana de $0.62 \mathrm{~mm}$. Hasta 8 pacientes tenían un grosor menor de $1 \mathrm{~mm}$ (53.3\%) y sólo 2 pacientes con MN mostraron una profundidad mayor de $2 \mathrm{~mm}$ (13\%). Hay que destacar que 2 $(40 \%)$ de los 5 melanomas ungueales eran malformaciones in situ, lo que representa un pronóstico excelente. Este grosor medio de $1.45 \mathrm{~mm}$ es inferior a la mitad de la media de otros estudios españoles, de 3.07 a $5.45 \mathrm{~mm}^{9,18-20} \mathrm{y}$, por supuesto, muy inferior a los $8.3 \mathrm{~mm}$ de un estudio brasileño ${ }^{15}$. Debe mencionarse también que 10 pacientes (66.7\%) de la muestra tenían estadios bajos (0-I). Tan sólo hubo un $33.3 \%$ de pacientes (5) con ulceración, lo que empeora el pronostico ${ }^{4,8}$. De los 11 casos con información 
de mitosis, se identificó a 6 pacientes con $<1 / \mathrm{mm}^{2}$ (54.5\%), lo que representa un estadio tumoral más bajo ${ }^{8}$. Por último, la BSGC fue positiva sólo en el $27.3 \%$ en los 11 pacientes en los que se realizó. Esta prueba proporciona información pronostica significativa y confirma que la supervivencia general es inferior en aquellos MA con una BSGC positiva respecto de los individuos en que es negativa ${ }^{5,8}$.

La localización del MA en este estudio sólo fue la superficie plantar $(66.7 \%)$ y la ungueal $(33.7 \%)$, sin observar ninguno en las palmas de las manos. En apariencia, el MA es mucho más raro en la superficie palmar, hasta de $8.6 \%$ de MA en algunas series ${ }^{18} y$ sólo de $1.7 \%$ en otras ${ }^{15}$. Algunos estudios refieren que la mayor parte de los melanomas de los pies se identifica en las zonas de apoyo, lo que sugiere que la presión tiene cierta función etiopatogénica, quizás en relación con los traumatismos repetidos ${ }^{10,18}$.

En este estudio se observó un grosor menor en los melanomas de uñas respecto de los de los pies, con una diferencia estadísticamente significativa, lo que indicaría un mejor pronóstico en los MU. Además, se reconocieron dos melanomas in situ en las uñas de las manos. Es posible que se deba a la mayor facilidad de reconocer las lesiones pigmentadas en las uñas que en las plantas de los pies y que, además, sea también más fácil en las uñas de las manos que en las de los pies.

Estos datos confirman que los MA suelen aparecer en personas adultas. La edad media en el diagnóstico fue de 58.87 años, que es similar a la de otros estudios $^{2,9}$. Sin embargo, debe considerarse que hubo dos pacientes femeninas con menos de 30 años (13.3\%), lo que indica la posibilidad de su aparición en personas jóvenes.

Aunque algunos especialistas señalan que el MA es más frecuente en mujeres ${ }^{7,18}$, los autores no encontraron diferencias genéricas, al igual que la mayoría de estudios $^{2,20}$. Es posible que las mujeres detecten antes los melanomas ${ }^{18}$. Además, tampoco se identificaron diferencias significativas en el estudio estadístico entre los dos géneros.

En este estudio, el MU comprendió el $33.3 \%$ de los MA, un porcentaje que es similar al de otros estudios que informan que el $30 \%$ a $35 \%$ de los melanomas acrales fueron ungueales ${ }^{3,4}$. Los cinco pacientes con MU se localizaron en los primeros dedos, el $40 \%$ en las manos y el $60 \%$ en los pies, lo que coincide con los estudios previos que registran una localización más frecuente en los primeros dedos ${ }^{18,19}$. No es claro si esta frecuencia aumentada se debe a una mayor predisposición a los traumatismos o a un mayor tamaño de la matriz ungueal que, en proporción, facilitaría su desarrollo.
Los melanomas ungueales de los dedos de los pies suelen mostrar ulceración más a menudo que los de las manos y tienen un mayor índice de Breslow y una mayor probabilidad de invasión ganglionar al diagnóstico. La explicación más plausible es que las lesiones localizadas en los dedos de las manos son más visibles y, en consecuencia, más reconocibles por los pacientes, lo que supone una consulta médica más temprana en comparación con las localizadas en los dedos de los pies ${ }^{18,19}$.

El tipo histopatológico más común de este estudio fue el MLA (73.3\%). Aunque el MLA es el tipo histopatológico característico en las localizaciones palmoplantar y ungueal, también pueden observase otros subtipos, con tasas de supervivencia no significativamente diferentes en estos sitios acrales ${ }^{6,7}$. En este estudio representaron el $26.6 \%$ (2 MES y $2 \mathrm{MN}$ ), lo que destaca que la media del grosor de Breslow fue más baja en los MLA y MES que en los MN. Un estudio español publicó que el grosor de Breslow fue mayor del doble en los MLA (3.5 mm) que en los MES $(1.4 \mathrm{~mm})$, independientemente del de los $\mathrm{MN}(4.9 \mathrm{~mm})^{9}$.

Se ha informado que la mortalidad específica entre los pacientes con MA se relaciona con un aumento del grosor del tumor y un estadio tumoral más avanzado al diagnóstico, pero no con el grupo étnico de los individuos. Los tumores con una profundidad de Breslow $>2 \mathrm{~mm}$ se acompañan de una menor supervivencia específica de melanoma. El peor acceso a la atención sanitaria puede explicar los resultados de supervivencia menos favorables de los pacientes de color oscuro?

Las supervivencias en este estudio han sido muy altas, del $92.3 \%$ al año y del $73.8 \%$ a los cinco años. Esto puede guardar relación con el hecho de que, según las aseveraciones de Asgari, et al. ${ }^{7}$, tan sólo el $13.3 \%$ de estos pacientes tenía un Breslow $>2 \mathrm{~mm}$ y el $66.7 \%$ mostró estadios bajos (0-I). Estos clínicos no encontraron diferencias en la supervivencia entre los dos géneros, ni los autores de este estudio, en función del sexo, la localización o el subtipo histopatológico.

\section{Conclusión}

Esta serie de pacientes ha presentado en general una relación de parámetros clínico-histopatológicos con un pronóstico bueno o aceptable. Debe destacarse que esos parámetros pronósticos han sido mejores en los melanomas de uñas que en los de las plantas de los pies. Puede ser que sea más fácil reconocer las lesiones pigmentadas en las uñas que en las plantas 
de los pies y que también lo sea en las uñas de las manos que en las de los pies.

\section{Conflicto de intereses}

Los autores confirman que no existe conflicto de intereses.

\section{Responsabilidades éticas}

Protección de personas y animales. Los autores declaran que para esta investigación no se han realizado experimentos en seres humanos ni en animales.

Confidencialidad de los datos. Los autores declaran que han seguido los protocolos de su centro de trabajo sobre la publicación de datos de pacientes.

Derecho a la privacidad y consentimiento informado. Los autores declaran que en este artículo no aparecen datos de pacientes.

\section{Bibliografía}

1. Elder DE, Bastian BC, Cree IA, Massi D, Scolyer RA. The 2018 World Health Organization Classification of Cutaneous, Mucosal, and Uveal Melanoma: detailed analysis of 9 distinct subtypes defined by their evolutionary pathway. Arch Pathol Lab Med. 2020;144:500-22.

2. Desai A, Ugorji R, Khachemoune A. Acral melanoma foot lesions. Part 1: epidemiology, aetiology, and molecular pathology. Clin Exp Dermatol. 2017;42:845-8

3. Desai A, Ugorji R, Khachemoune A. Acral melanoma foot lesions. Part 2: clinical presentation, diagnosis, and management. Clin Exp Dermatol. 2018;43:117-23.
4. Dika E, Patrizi A, Fanti PA, Chessa MA, Reggiani C, Barisani A, et al. The prognosis of nail apparatus melanoma: 20 years of experience from a single institute. Dermatology. 2016;232:177-84.

5. Criscito MC, Stein JA. Improving the diagnosis and treatment of acral melanocytic lesions. Melanoma Manag. 2017;4:113-23.

6. Merkel EA, Gerami P. Malignant melanoma of sun-protected sites: a review of clinical, histological, and molecular features. Lab Investig. 2017;97:630-5.

7. Asgari MM, Shen L, Sokil MM, Yeh I, Jorgenson E. Prognostic factors and survival in acral lentiginous melanoma. Br J Dermatol. 2017;177:428-35.

8. Keung EZ, Gershenwald JE. The eighth edition American Joint Committee on Cancer (AJCC) melanoma staging system: implications for melanoma treatment and care. Expert Rev Anticancer Ther. 2018;18:775-84.

9. Carrera C, Gual A, Díaz A, Puig-Butillé JA, Noguès S, Vilalta A, et al. Prognostic role of the histological subtype of melanoma on the hands and feet in caucasians. Melanoma Res. 2017;27:315-20.

10. Jung HJ, Kweon SS, Lee JB, Lee SC, Yun SJ. A clinicopathologic analysis of 177 acral melanomas in koreans: relevance of spreading pattern and physical stress. JAMA Dermatol. 2013;149:1281-8.

11. Fujisawa Y, Yoshikawa S, Minagawa A. Takenouchi T, Yokota K, Uchi H, et al. Clinical and histopathological characteristics and survival analysis of 4594 japanese patients with melanoma. Cancer Med. 2019;8:2146-56.

12. De la Fuente A, Ocampo-Candiani J. Melanoma Cutáneo. Gac Med Méx. 2010;146:126-35.

13. Pozzobon F, Acosta A, Carreño A, Fierro E. Características del melanoma cutáneo primario en el Instituto Nacional de Cancerología 2006-2010. Rev Colomb Cancerol. 2013;17:111-8.

14. Valenzuela F, Yagnam M, Zemelman V, Morales C, Valenzuela CY. Melanoma acral: a propósito de un caso. Rev Hosp Clin Univ Chile. 2013;24:286-9.

15. Zegarra del Carpio R. Situación del melanoma maligno cutáneo en el Hospital Militar Central de Lima 1985-2007. Dermatol Perú. 2008;18:267-83.

16. Nunes LF, Quintella Mendes GL, Koifman RJ. Acral melanoma: a retrospective cohort from the Brazilian National Cancer Institute (INCA). Melanoma Res. 2018;28:458-64.

17. Loria DI, González A, Latorre C. Epidemiología del melanoma cutáneo en Argentina: análisis del Registro Argentino de Melanoma Cutáneo. Dermatol Argent. 2010;16:39-45.

18. Pereda C, Traves V, Requena C, Serra-Guillén C, Serra-Guillén C, Llombart B, et al. Estudio descriptivo de la presentación clínica del melanoma lentiginoso acral. Actas Dermosifiliogr. 2013;104:220-6.

19. Talavera-Belmonte A, Bonfill-Ortí M, Martínez-Molina L, Fornons-Servent R, Bauer-Alonso A, Ferreres-Riera JR, et al. Melanoma subungueal: estudio descriptivo de 34 pacientes. Actas Dermosifiliogr. 2018;109:801-6.

20. Mazuecos J, Herrera A, Fonseca E, Martínez L, Camacho F. Estudio retrospectivo del melanoma lentiginoso acral del pie durante las dos últimas décadas. Podología Clínica. 2002;3:41-7. 